\title{
1 CRISPR-Cas systems in Fusobacterium: An untapped genetic frontier
}

\section{Ariana Umaña ${ }^{1}$, Daniel J. Slade ${ }^{1, \#}$}

$3{ }^{1}$ Department of Biochemistry, Virginia Polytechnic Institute and State University, Blacksburg, VA, USA.

4 \# To whom correspondence should be addressed: Dr. Daniel J. Slade, Department of Biochemistry, Virginia

5 Polytechnic Institute and State University, Blacksburg, VA 24061. Telephone: +1 (540) 231-2842. Email:

\section{$6 \quad$ dslade@vt.edu}

A Umaña: $\quad$ 0000-0002-1941-8656

DJ Slade: $\quad$ 0000-0001-5634-7220

\section{ABSTRACT}

Fusobacterium nucleatum has recently received significant attention for its strong connection with the acceleration and gravity of multiple cancers (e.g., colorectal, pancreatic, esophageal).

However, our understanding of the molecular mechanisms that drive infection by this

'oncomicrobe' have been hindered by a lack of universal genetic tools. Herein we report a global immune systems including Cas13c, and detailed report of the proteins, spacer/repeat loci, transactivating CRISPR RNA (tracrRNA), and CRISPR RNA (cRNA) from a Type II-A CRISPR-Cas9 system. Since most Fusobacterium are currently genetically intractable, this CRISPR-Cas

bioinformatic roadmap could be used to build new genome editing and transcriptional tuning tools to characterize an increasingly important genus of human opportunistic-pathogens connected to the onset, progression, and severity of cancer. 


\section{INTRODUCTION}

Fusobacteria are anaerobic Gram-negative bacteria, with the most well studied human and

livestock opportunistic pathogens of this genus being F. nucleatum and F. necrophorum. Due to robust restriction modification systems that hinder genome manipulation, Fusobacterium genetics have classically been considered nearly impossible, resulting in few bacterial strains that can be genetically modified for detailed mechanistic studies. Despite recent advances in F. nucleatum genetic tools, mainly in the strain F. nucleatum subsp. nucleatum ATCC 23726 , all other species outside of $F$. nucleatum have nearly fallen into the category of genetically intractable ${ }^{1-3}$, with the exception being a recent study highlighting a conjugation method for genetic manipulation of $F$. necrophorum ${ }^{4}$.

Recent advances in our understanding of Clustered regularly interspaced short palindromic repeats-CRISPR-associated proteins (CRISPR-Cas) systems have been exploited by molecular biologists to allow for efficient and robust genome editing in both prokaryotic and eukaryotic systems ${ }^{5-7}$. CRISPR and Cas proteins comprise the basis of adaptive immunity in bacteria and archaea ${ }^{8}$. CRISPR-Cas systems identify and cleave non-host DNA and RNA (viruses and plasmids), and deposit a $\sim 30$ bp segment into its host genome at defined CRISPR loci (host DNA repeats and foreign protospacer, or 'spacer' DNA) to provide organism- and sequence-specific defenses ${ }^{9}$. During repeat infections, CRISPR-Cas complexes recognize these specific sequences and rapidly clear the invading DNA through active nucleotide cleavage ${ }^{10}$. CRISPR systems have been characterized in $87 \%$ of archaea and $47 \%$ of bacteria and have been proposed to proliferate through horizontal gene transfer across bacteria as a basis of adaptive immunity ${ }^{9}$. 
CRISPR arrays are divided into three main categories and 16 distinct subcategories based on the cas genes encoded within bacteria and archaea ${ }^{11}$. However, the majority of genome editing studies have used the Cas9 protein from S. pyogenes ${ }^{11}$. While the use of S. pyogenes Cas 9 in prokaryotic and eukaryotic systems has been achieved, our goal was to characterize

Fusobacterium CRISPR-Cas proteins to determine if these proteins will be more efficient for future gene modulation studies in an AT rich genus of bacteria. The discovery and characterization of native Class 2 effectors in Fusobacterium will potentially enhance the application and development of CRISPR systems for genome engineering. Herein, we report a comprehensive bioinformatic characterization of multiple Fusobacterium CRISPR-Cas adaptive immune systems, focusing on

Cas9 and the newly described Cas13c present in F. necrophorum subsp. funduliforme 1_1_36S.

This data, coupled with a recent study characterizing an active CRISPR system in the strain $F$. nucleatum subspecies nucleatum ATCC $25586^{12}$, provides hope that the development of new CRISPR-based genetic tools will enhance our understanding of fusobacterial physiology and pathogenesis.

\section{MATERIALS AND METHODS}

Use of genomic information for bioinformatic analysis and identification of CRISPR-Cas systems in eight Fusobacterium strains.

Fusobacterium genomes (F. nucleatum subsp. nucleatum ATCC 23726 (GCA_003019785.1), F. nucleatum subsp. nucleatum ATCC 25586 (GCA_003019295.1), F. necrophorum subsp. funduliforme 1_1_36S (GCA_003019715.1), F. varium 27725 (GCA_003019655.1), F. ulcerans 49185 (GCA_003019675.1), F. mortiferum 9817 (GCA_003019315.1), F. gonidiaformans 25563 (GCA_003019695.1), and F. periodonticum 2_1_31 (GCA_003019755.1) were used to extract all 
sequences to analyze using the CRISPROne web server ${ }^{13}$ and CRISPRCasFinder ${ }^{14}$.

CRISPROne and CRISPRCasFinder were used to predict all CRISPR-Cas associated elements as well as the repeat arrays.

\section{Bioinformatic analysis of CRISPR-Cas Class 2 systems and identification of effector domain}

The genome sequence from F. necrophorum subsp. funduliforme 1_1_36S (GCA_003019715.1) was used to predict the open reading frame for the CRISPR-Cas systems. An open reading frame of 1337 amino acids for Cas 9 and 1121 amino acids for Cas13c was identified using the web server pHMMER ${ }^{15}$ and stand-alone HMMER software package, respectively.

\section{Phylogenetic Tree construction}

Full CRISPR-Cas phylogenetic analysis was created using the micropan ${ }^{16}$ plugin in Rstudio by using only CRISPR associated proteins to build the tree. The gene maps in Figure 1B were created using the gggenes ${ }^{17}$ plugin in Rstudio. Additional phylogenetic trees for Cas 9 and Cas $13 \mathrm{c}$ were created in Geneious 9.18 utilizing the multiple sequence alignment from SMARTBLAST and adapted in Affinity Designer.

\section{Structure Prediction}

The structure prediction of Cas9 from Fusobacterium necrophorum subsp. necrophorum 1_1_36S was performed in the web-based suite Phyre2 ${ }^{18}$. CRISPR RNA structure prediction was done using NUPACK ${ }^{19}$.

\section{RESULTS}




\section{Comparative Analysis of Fusobacterium CRISPR-Cas systems}

A total of eight Fusobacterium genome sequences were utilized for bioinformatic comparative analysis and the composition, occurrence, and diversity of CRISPR-Cas systems. Presently, there are two classes and six types of CRISPR-Cas systems based in the organization of their effector modules. A majority of the identified CRISPR-Cas systems in Fusobacterium belong to Class 1 ( $77 \%$ of CRISPR-Cas systems in the eight genomes) and interestingly, only $F$. necrophorum susbp. funduliforme 1_1_36S and F. ulcerans 49185 encode for Class 2 systems (23\% of CRISPR-Cas systems in the eight genomes). The overall analysis of CRISPR-Cas systems is shown in Table S1.

To depict the correlation among Fusobacterium species and strains, a CRISPR-Cas system-based phylogenetic tree was constructed. As shown in Figure 1A, the eight Fusobacterium strains are divided into five distinct clades according to their predicted CRISPR-Cas systems. Interestingly, F. nucleatum ATCC 23726 (Lineage 1) clades with F. gonidiaformans 25563 (Lineage 3) and F. periodonticum 2_1_31 (Lineage 1) clades with F. necrophorum subsp. funduliforme 1_1_36S (Lineage 3).

The rapid evolution of CRISPR systems explains the diversity of systems from highly similar strains. For instance, F. nucleatum ATCC 23726 and F. nucleatum ATCC 25586 are the closest phylogenetically when using whole genome phylogenetic analysis, yet as seen in Figure 1A, they are far more divergent in their CRISPR loci. Additionally, the analysis revealed that $F$. mortiferum 9817 CRISPR-Cas systems have less conservation when compared to the other Fusobacterium strains. 


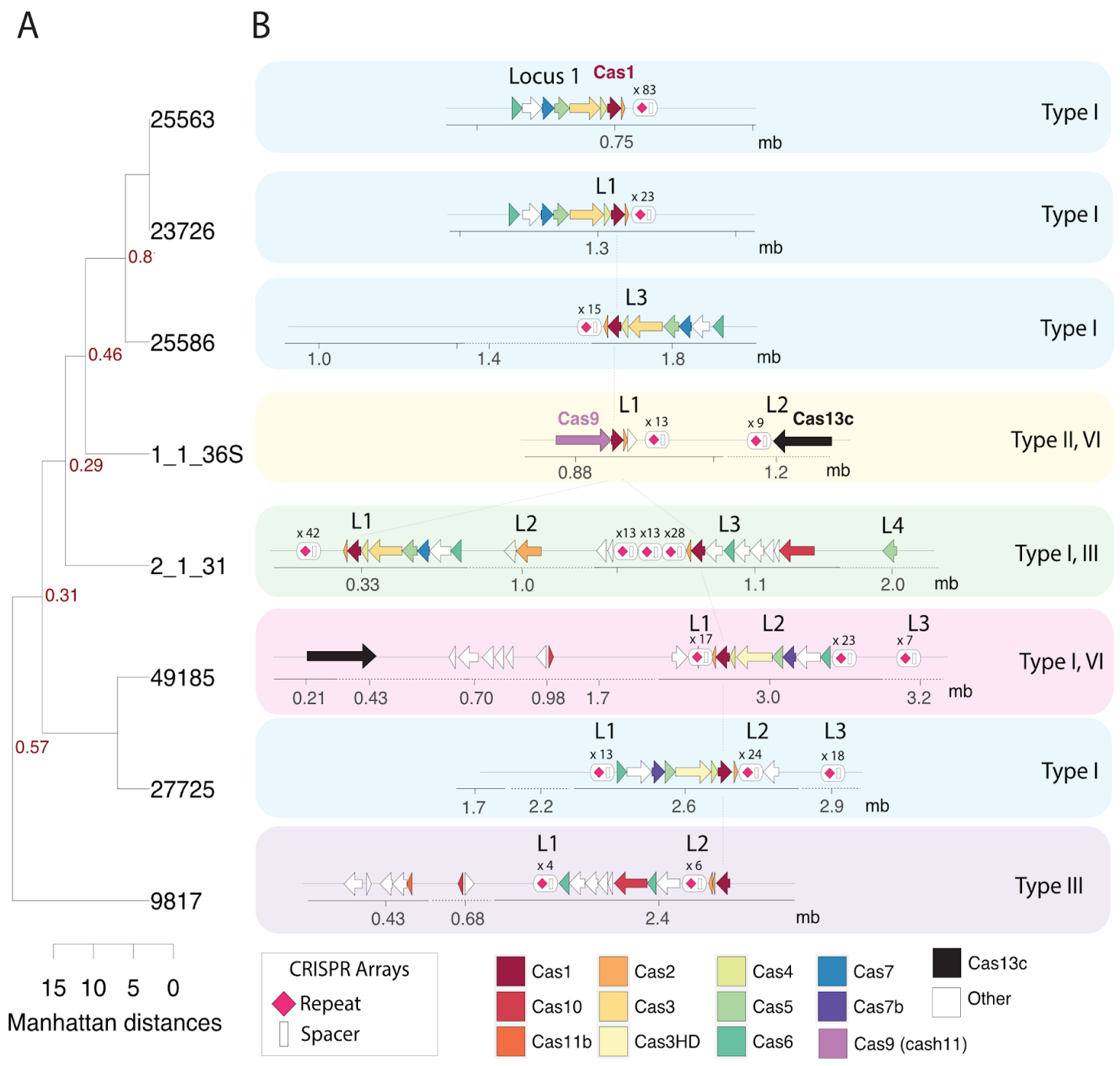

Figure 1. CRISPR-Cas systems in Fusobacterium. A. CRISPR-Cas phylogenetic tree of eight

Fusobacterium strains. B. A total of eight Fusobacterium strains were utilized for bioinformatic analysis and identification of CRISPR-Cas systems. A majority of the identified CRISPR-Cas systems in Fusobacterium belong to Class 1 and only F. necrophorum susbp. funduliforme 1_1_36S and F. ulcerans 49185 encode for Class 2 systems.

Estimation of spacer numbers and sequences among Fusobacterium species exhibited substantial quantity of predicted spacers varies among the strains, ranging from 4 to 83 , demonstrating the biological heterogeneity of Fusobacterium strains that encode an adaptive defense mechanism. Type I-B presented a higher number of spacers when compared to the other CRISPR-Cas system types. 


\section{Classification of CRISPR-Cas systems in Fusobacterium}

To determine the classification and assortment of CRISPR-Cas systems across the eight

Fusobacterium strains, we utilized the CRISPRCasFinder and CRISPROne web server that

enabled the discovery of direct repeat consensus sequences within a genome as well as the

characterization of related spacers and cas genes ${ }^{13,14}$. This analysis detected a total of 13

CRISPR-Cas systems belonging to both Class 1 (Type I, Type III and Type IV,) and Class 2 (Type

II, Type VI): six Type I (54.54\%), one Type II (9.09\%), two Type III (18.18\%), and two Type VI

(18.18\%) while no Type IV or V was identified. Therefore, we conclude that Type I is the

predominant CRISPR-Cas system among the studied Fusobacterium strains. Detailed illustrations

of the CRISPR-Cas systems identified among the strains analyzed in this study are summarized in

Figure 1B. F. nucleatum ATCC 23726, F. nucleatum ATCC 25586, F. gonidiaformans 25563 and

F. periodonticum 2_1_31 Type I-B is a Class 1 CRISPR-Cas loci that includes full gene sets to achieve adaptive immunity: cas1, cas2 and cas4 for spacer integration or adaptation, cas6 for crRNA-processing and cas3, cas5, cas7, and cas8 for interference. Both $F$. ulcerans 49185 and $F$. varium 27725 include a nearly complete Type I loci, but they do not encode for the cas 8 gene. Additionally, most CRISPRs of Type I-B CRISPR-Cas systems discovered in this analysis were encoded downstream of the cas 2 gene and/or upstream of the cas 6 gene. Worth noting, $F$. nucleatum ATCC 23726 and F. gonidiaformans 25563 presented an inverted loci composition of the Type I-B CRISPR-Cas systems identified (Figure 1B).

Furthermore, in F. periodonticum 2_1_31, Type I and Type III systems appear to combine as they often coexist ${ }^{20}$. Type III systems have been divided into four major subtypes (III-A to D) 
51 interference steps was identified in F. periodonticum 2_1_31. Additionally, Type III systems are

52 found to frequently encode for accessory genes near the core cas gene cluster.

Analysis of a F. necrophorum Type II CRISPR-Cas system and identification of putative

The only Type II-A CRISPR-Cas system identified in our analyzed Fusobacterium strains belonged

A

F. necrophorum 1_1_36S
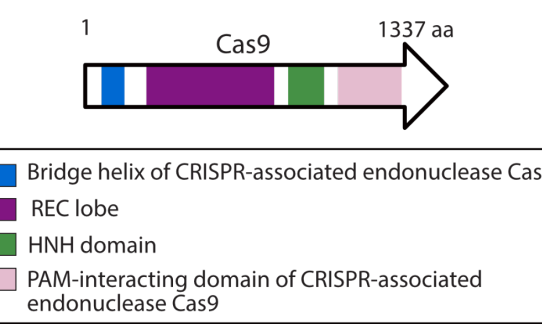

Bridge helix of CRISPR-associated endonuclease Cas REC lobe

HNH domain

PAM-interacting domain of CRISPR-associated endonuclease Cas 9

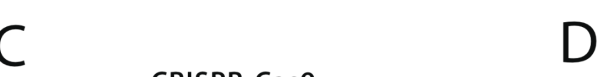

CRISPR-Cas 9

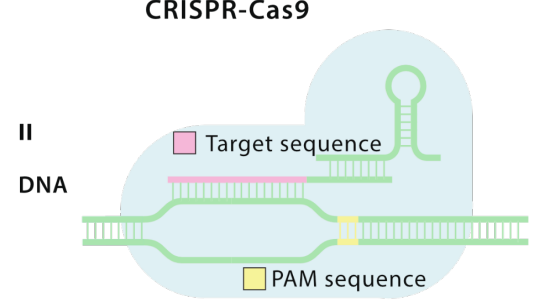

S. pneumoniae

Neisseria

Leptotrichia

Crenarcheaeota archea

Fusobacterium

F. necrophorum 1_1_36S

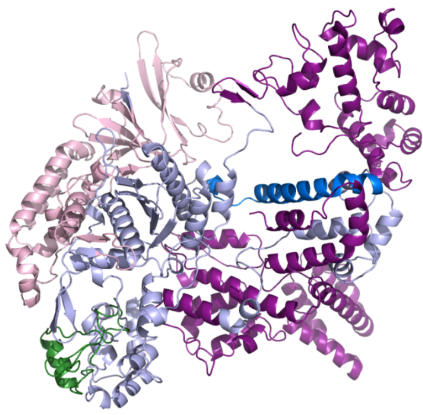

Type II Cas9 RNA-guided

Type II Cas9 RNA-guided

Figure 2. Class II CRISPR-Cas9 system in F. necrophorum subsp. funduliforme 1_1_36S. with domains color coordinated with section A. C. Schematic of CRISPR-Cas9 in F. necrophorum subsp. funduliforme 1_1_36S. D. SMART-BLAST phylogenetic analysis of Cas9. 
The Type II CRISPR-Cas systems function through the assembly of an effector module consisting of a single Cas9 protein, a crRNA and a tracrRNA. Cas9 is 1337 amino acids in size. A previous genome assembly of $F$. necrophorum subsp. funduliforme 1_1_36S had this protein as 329 amino acids, proving that complete and accurate genome sequences found in FusoPortal were key for identifying the proper open reading frames of these proteins ${ }^{24}$. The predicted structure of Cas 9

(Figure 2B) resembles the characteristic bi-lobed architecture from previously characterized Cas 9 proteins, with the RuvC and HNH domains for nucleic acid cleavage (Figure 2A). In addition to Cas9, recognition and targeting of DNA is dependent on the crRNA and tracrRNA complex ${ }^{25}$. For this reason, we characterized the tracrRNA element of the Type II CRISPR-Cas system in F. necrophorum subsp. funduliforme 1_1_36S. The first nucleotides predicted in the nexus include adenine residues that are known to be highly conserved among tracrRNAs of Type II systems as shown in Figure $3 \mathbf{A}^{25}$ and the bulge interacts through a specific side-chain with the Rec1 domain or lobe through specific side-chain ${ }^{26}$. Both bulge and nexus in tracrRNA are required for DNA cleavage, and the spacer sequence detailed in Figure 3B will determine the location of Cas 9 endonucleolytic cleavage ${ }^{25}$. Finally, the stems interact with Cas 9 mainly through sequence-independent interactions with the phosphate backbone ${ }^{25}$.

revealed a conservation within the Fusobacterium genus (Figure 2B). While the PAM sequence for F. necrophorum subsp. funduliforme 1_1_36S has not been experimentally characterized, the predicted PAM sequence for this Cas9 is NGG ${ }^{27}$; the same as used for the commonly used $S$. pyogenes Cas9. By contrast, the predicted PAM sequence for the Type I-B CRISPR-Cas systems from F. nucleatum ATCC 23726 is YNW ${ }^{27}$. Overall, a total of 3,427 spacers have been identified in 


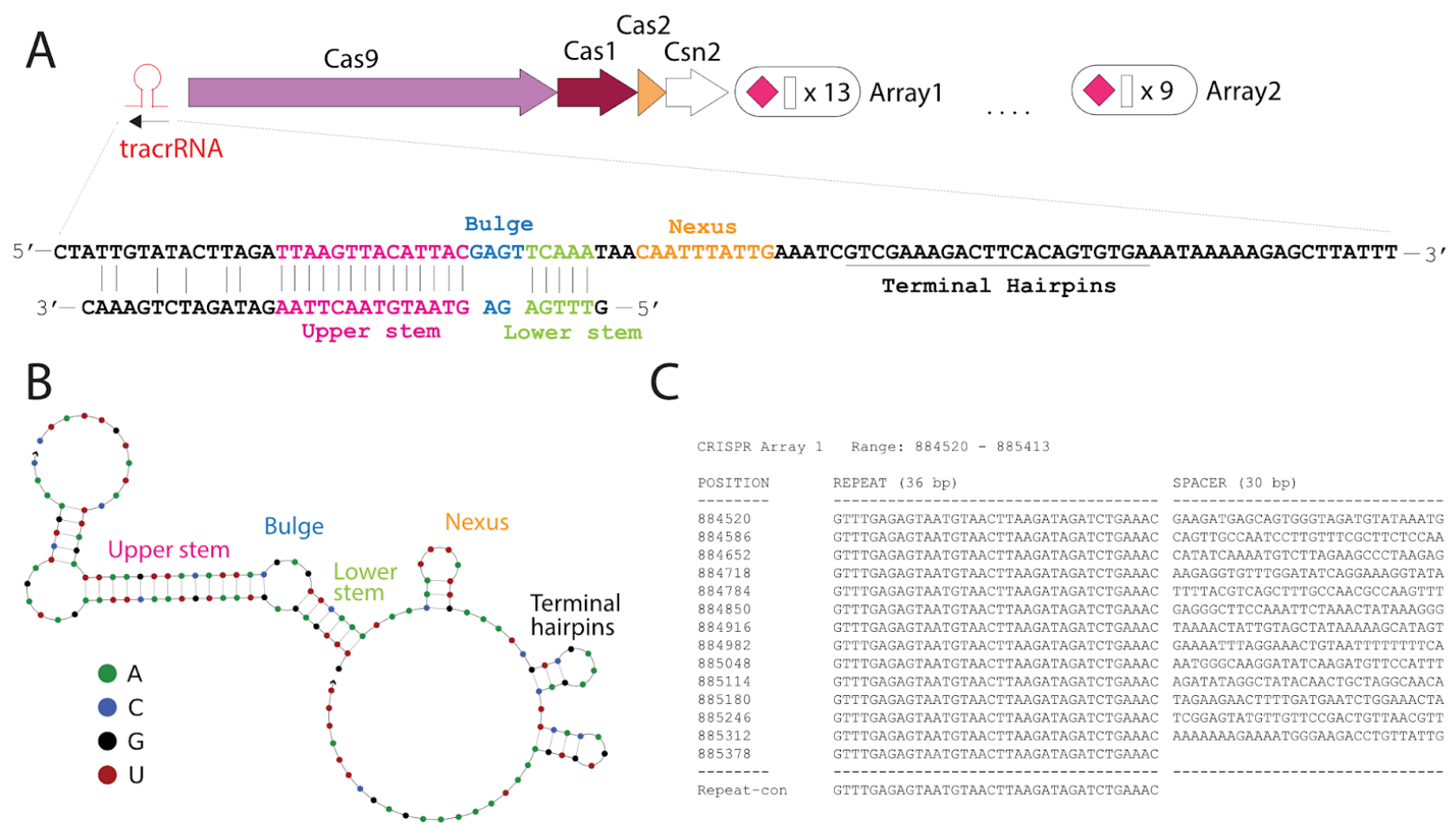

Figure 3. Characterization of CRISPR-Cas elements from $F$. necrophorum susbp. funduliforme 1_1_36S. A. Predicting the upstream Trans-activating CRISPR RNA (tracrRNA) of Cas9, coupled with the repeat region complementary to the tracrRNA. B. Prediction of the secondary structure of Cas 9 tracrRNA. C. Overview of CRISPR repeats (36 bp) and spacers (30 bp) for the Cas9 locus of $F$. necrophorum subsp. funduliforme 1_1_36S.

Fusobacteria with only 629 matching viral, intergenic or ORFs ${ }^{28}$. Utilizing viruSITE ${ }^{29}$, the integrated database for viral genomics, most of the 13 spacers of the CRISPR array from Fusobacterium subsp. funduliforme 1_1_36S Cas9 were identified as a potentially part of Synechococcus, Bacillus, Enterobacteria, or Aeromonas phage genomes.

Identification of the F. necrophorum subsp. funduliforme 1_1_36S and F. ulcerans 49185 Type IV CRISPR-Cas systems 
A

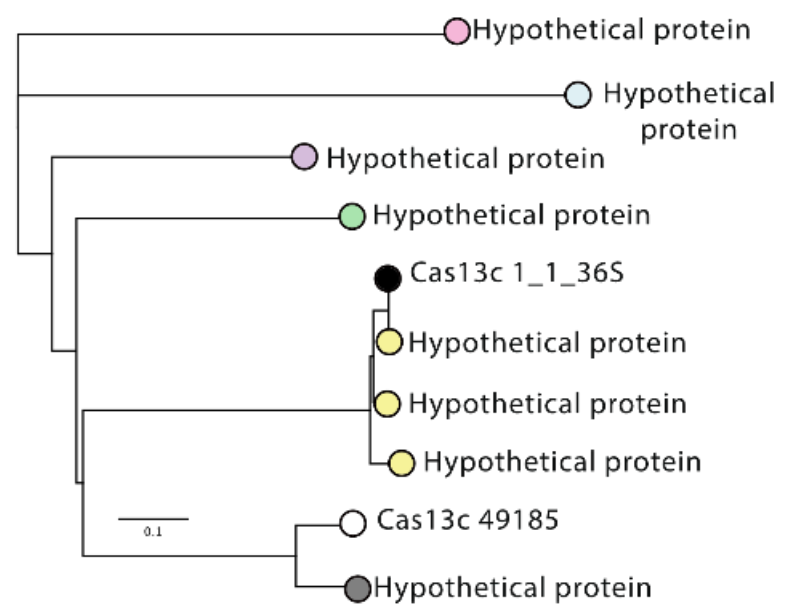

B

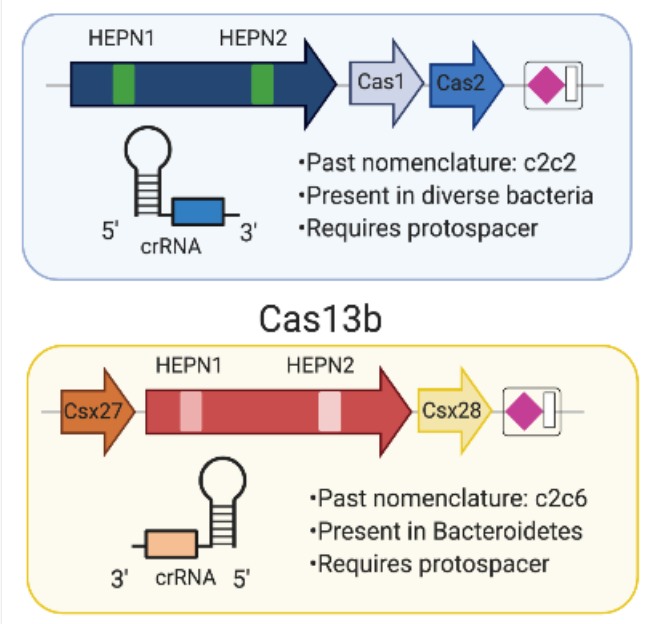

Cas $13 \mathrm{c}$

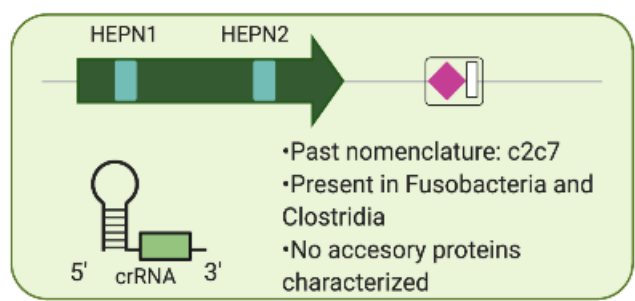

Cas13d

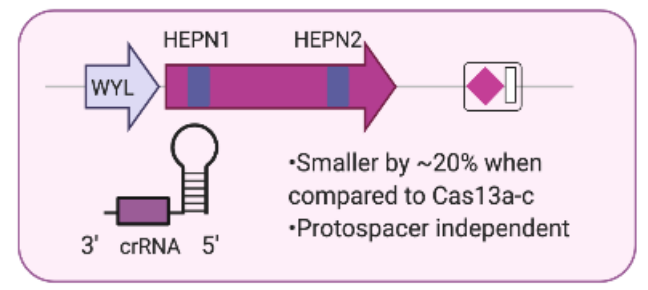

Figure 4. Analysis of Cas13c from a Type VI CRISPR-Cas system in F. necrophorum subsp.

funduliforme 1_1_36S. A. SMART-BLAST phylogenetic analysis of Cas13c. B. domain organization of the subsets of Cas 13 enzymes.

Finally, Type VI CRISPR-Cas systems are divided into three types due to their low sequence

similarity: VI-A, VI-B and VI-C. However, they all share the catalytic motif of the HEPN domain ${ }^{30}$.

The R-X4-H (Figure S1) motifs which are characteristic of HEPN-binding domains in both Cas13c

that potentially mediate RNA cleavage is highlighted in Figure $4^{31}$. F. necrophorum subsp.

:11 funduliforme 1_1_36S and F. ulcerans 49185 shared a $40.51 \%$ and $40.64 \%$ of identity when 
:12 compared to the previously identified Cas13c from $F$. perfoetens ${ }^{32}$. The Cas 13c from $F$. ulcerans

:13 is composed of 1117 amino acids while the Cas $13 c$ from $F$. necrophorum is 1137 amino acids,

!14 which is consistent with the average size for Cas $13 \mathrm{c}$ of approximately 1120 amino acids ${ }^{33}$.

:15 A phylogenetic analysis of bioinformatically predicted Cas13c uncovered the close

!16 phylogenetic relationship between Type VI effectors in Fusobacterium (Figure 4A). Finally, when

!17 compared to other Type VI effectors Cas13c are the least studied and no accessory proteins have :18 been identified like the other recently discovered Type VI systems.

\section{DISCUSSION}

As CRISPR loci consist of host repeats and segments of foreign DNA extracted from invading

organisms and mobile genetic elements, these repetitive stretches of DNA have proven difficult to resolve using short read DNA technologies that are commonly used to sequence bacterial

genomes. The emergence of long-read DNA technologies from Oxford Nanopore and Pacific

Biosciences has opened the door for the completion of fragmented genomes; many of which are

likely incomplete due to the inability to resolve repeat regions. Previous attempts to determine the conformation, quantity, and diversity of CRISPR-Cas systems in Fusobacterium were exceptionally challenging because the complete genome sequencing information was incomplete, restricted, and incorrect. Complete and accurate genome sequences as the ones provided by FusoPortal are crucial for the analysis of these systems as well as the recombinant production of Cas proteins, or incorporation into a plasmid or chromosome; potentially leading to the expansion of Fusobacterium genetic toolkit 24,34 .

A large percentage of bacteria harbor adaptive defense systems known as CRISPR-Cas systems, the analysis of CRISPR-Cas systems in Fusobacterium uncovered Class 1 and Class 2 systems. 
Class 2 systems are less common within prokaryotes when compared to Class 1 systems, at most

$10 \%$ of CRISPR-Cas systems within sequenced genomes belong to Class 2 systems ${ }^{35}$. For that

reason, most of the identified CRISPR-Cas systems in Fusobacterium belong to Class 1 and

interestingly, only F. necrophorum subsp. funduliforme 1_1_36S and F. varium 49185 encode for

Class 2 systems which is similar to the CRISPR-Cas systems identified in Leptotrichia, which

belongs to Fusobacterium genus, where they are unevenly distributed ${ }^{36}$.

Type I CRISPR-Cas systems were found to be the most abundant in the eight Fusobacterium

strains, which often coexist with Type III CRISPR-Cas systems as shown in F. periodonticum

2_1_31. It is suspected that Type I systems complement Type III systems functionally ${ }^{37}$.

Nonetheless, whereas Type I systems especially degrade dsDNA, Type III systems target ssDNA, dsDNA, and ssRNA ${ }^{37}$. CRISPR mediates adaptive immunity by ensuing three steps. Particularly, most of Fusobacterium presented a complete operon of Type I-B CRISPR-Cas systems which is required to achieve adaptive immunity. First, the adaption step by Cas1, Cas 2 and Cas4 requires the acquisition and inclusion of short segments of exogenous nucleotides into CRISPR loci; then crRNA-processing by Cas 6 where the crRNA expression and maturation take place, and the last varium 27725 which lack the signature cas 8 gene, the interference step could be defective as it has been demonstrated that Cas8 plays a key role in targeting cascade to invader DNA ${ }^{39}$. Most Type I-B CRISPR-Cas systems in the analyzed eight strains were encoded downstream of the cas2 gene and/or upstream of the cas6 gene which is in accordance with a CRISPR-Cas system present in the anaerobic rod Clostridium thermocellum ${ }^{40}$. 
Type III-A CRISPR-Cas adaptive immune systems are composed of a protein-RNA complex

21. The complete loci in F. periodonticum 2_1_31 is constituted of five proteins and additional

proteins that contain the Csm targeting complex Cas10/Csm1, Csm2-Csm5 and the Csm6

ribonuclease while F. mortiferum 9817 lacks Csm1 and Csm6 which could result in a non-

functional CRISPR-Cas system. The role of these accessory genes may be related to modulation,

complementation, or extension of these systems $22,37,41$.

To determine how CRISPR-Cas systems will function the Type, Class and PAM recognition

of the endonucleases needs to be characterized. For the Type II-A CRISPR-Cas system in F.

necrophorum subsp. necrophorum 1_1_36S the PAM sequence was previously predicted as NGG

and we further characterized its tracrRNA and spacer composition ${ }^{27}$. This system could be utilized

for genome modification in Fusobacterium strains (Figure 5), however distinct strains of an

organism encode as shown in this study, different elements to their CRISPR systems and

consequently, may have unique requirements for genetic editing.

The most conserved trait in Type VI CRISPR-Cas is the HEPN domain ${ }^{30}$. Interestingly enough,

Type III systems possess RNase activity through the HEPN domain contained in the Csm6 and

Csx1, although their role in this specific system remains to be studied ${ }^{42}$. The first report of a Type

VI system bioinformatically identified in Fusobacterium belonged to $F$. perfoetens ${ }^{32}$, a strain that

was found in children with diarrhea, nursing infants and in feces of a piglet. Additionally, CRISPR-

Cas V1-C systems were only found in Clostridium and Fusobacterium ${ }^{30}$.

The subtypes in the VI systems are based in the HEPN domains which are highly variable, the most widely studied being Type VI-A (Figure 4) ${ }^{11}$. The HEPN domains are found frequently in various defense systems ${ }^{30}$, in Cas13d proteins, this domain has been found to orchestrate 
HEPN domains identified in Cas13c from F. necrophorum subsp. funduliforme 1_1_36S and F.

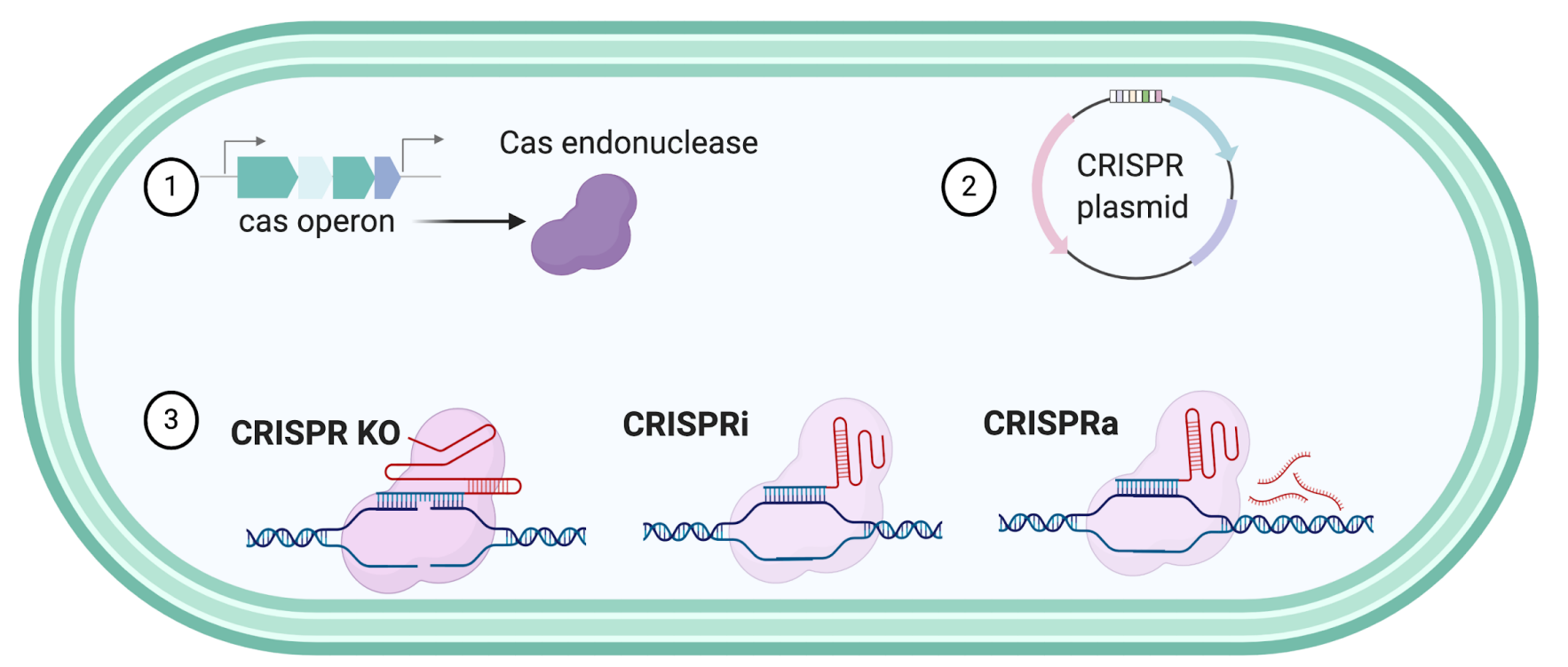

Figure 5. The use of native CRISPR-Cas systems as genetic tools could lead to the development of powerful tools. These tools could improve genome editing of Fusobacterium by creating deletion systems, characterization of essential genes through the CRISPRi, or the overexpression proteins using CRISPRa.

Additionally, CRISPR-Cas spacers represent a successful defense mechanism from past intruders, and the conserved spacers reflect the evolutionary phylogeny or conservation of species and strains ${ }^{36}$. In the studied Fusobacterium strains, the spacers were not similar within them and the number variability suggests the biological heterogeneity of Fusobacterium species that encode a CRISPR-Cas system as a defense mechanism and consequently, for modulation of immunity ${ }^{36}$. Higher number of spacers was observed in Type I-B which coincides with the fact that Type I-B, IIC and III-like CRISPR-Cas systems overall contain an increased number of spacers when compared to Type III-A, III-D, and VI-A.

In conclusion, the entirety of the Fusobacterium strains studied have endogenous systems that could be exploited as genetic tools for genomic engineering. The development of a CRISPR- 
.97 Cas gene editing tool in Fusobacterium requires the description and detailed examination of the

!98 Type/Class of CRISPR array and identifying the PAM recognition sequence. CRISPR-Cas systems are a good candidate for genetic engineering since they depend on the endogenous systems that provide existing components and it is necessary to expand the genetic toolbox as Fusobacterium only has a few genetic systems described ${ }^{1,2}$.

The utilization of bioinformatics has contributed to the continued discovery of novel CRISPR-Cas systems, such as Type V and Type VI CRISPR-Cas systems. As this bioinformatic study validates the presence of diverse CRISPR-Cas systems in Fusobacterium, we hypothesize that this technology could be used to more efficiently introduce gene interruptions, point mutations, and transcriptional control, through CRISPR interference (CRISPRi) and activation (CRISPRa)

(Figure 5) ${ }^{43}$. Furthermore, the CRISPR-Cas technologies and applications that have been developed are rapidly evolving and could enable countless novel applications ${ }^{32}$, which is of substantial impact as most strains of Fusobacterium do not contain native plasmids and the need to create broad host plasmids that allow for pan-genetics through CRISPR technologies is a priority for the field.

\section{Declaration of competing interests}

The authors declare no competing interests.

\section{Acknowledgments}

The authors would like to acknowledge Dr. Kira Marakova (NCBI) for the insightful information 
bioRxiv preprint doi: https://doi.org/10.1101/2022.01.21.477265; this version posted January 21, 2022. The copyright holder for this preprint (which was not certified by peer review) is the author/funder, who has granted bioRxiv a license to display the preprint in perpetuity. It is made available under aCC-BY-NC-ND 4.0 International license.

\title{
i59 SUPPLEMENTAL FIGURES
}

\author{
N-terminal HEPN domain
}

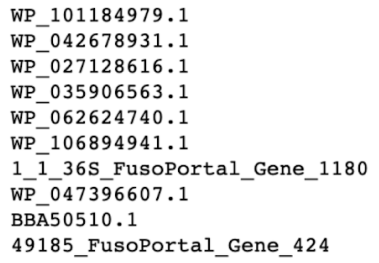

WP 101184979.1

WP_042678931.

WP_027128616.

WP 035906563 .

WP 062624740.1

WP_106894941.1

1_1_36S_FusoPortal_Gene_1180

WP_047396607.1

BBA50510.1

49185_FusoPortal_Gene_424

WP 101184979.1

WP 042678931.1

WP_027128616.

WP_035906563.1

WP 062624740 .

WP_106894941.1

1_1_36S_FusoPortal_Gene_1180

WP_ 047396607.1

BBA50510.1

49185_FusoPortal_Gene_424

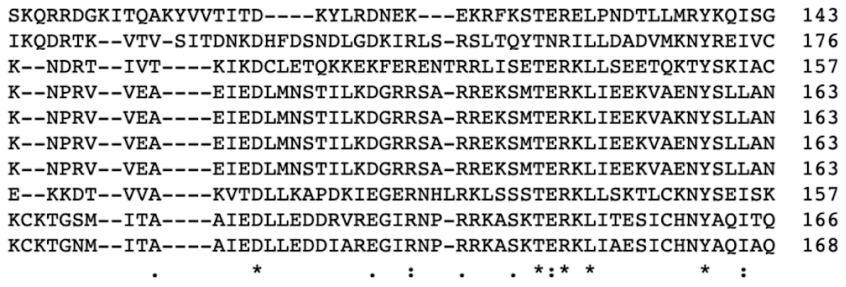

FDT--------LTSKDIYKIKRYIDFKNEMLFYFOFIEEFFSPLILPKGT---------NF- 186 SDSEKVDETINIDSOEIYKINRFLSYRSNMI IYYOMINNFLLHYDGEEDKGGNDSINLIN 236 CSPED------IDSVKIYKIKRYLAYRSNMLLFFSLINDIFVKGVVK--DN----GEEVG 205 CPMEE-------VDSIKIYKI KRFLTYRSNMLLYFASINSFLCEGIKG--KD------NETE 210 CPMEE------VDSIKIYKI KRFLTYRSNMLLYFASINSFLCEGIKG--KD------NETE 210 CPMEE-------VDSIKIYKIKRFLTYRSNMLLYFASINSFLCEGIKG--KD------NETE 210 CPMEE------VDSIKIYKIKRFLTYRSNMLLYFASINSFLCEGIKG--KD------NETE 210 TPIEE-------IDSIKIYKI KRFLNYRSNFLIYFALINDFLCAGVKE---------DDIN 202 CPVEE------IDAVKIYKVKRFLSYRSNMLLFFALINDFLCKNLKN--EK----GEKIN 214 CPVEE-------IDAVKIYKVKRFLSYRSNMLLFFALINDFLCKNLKN--KK-----GEKIN 216

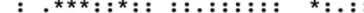

--YSLNIE---------ONKDKVVKY IVYRLNDDFKN--------------------- 212 EIWKYENKKNDEKE-------KIIERSYKS----------IEKSINQYILN-------- 270 EIWRIIDS---KEIDEKKTYDLLVENFKKRMSQEF INYKQSIENKIEKNTNKIKEIEQKL 262 EIWHLKDNDVRK--------EKVKENFKNKLIQSTENYNSSLKNQIEEKEKLLRKESK-- 260 EIWHLKDNDVRK--------EKVRENFKNKLIOSTENYNSSLKNOIEEKEKLLRKEFK-- 260 EIWHLKDNDVRK EIWHLKDWDVRKEIWHLKDNDVRK--------EKVKENFKNKLIQSTENYNSSLKNQIEEKEKLLRKESK-- 260 EVWLIQDKEHTAFLENR--IEKITDY IFDKLSKDIENKKNQFEKRIKKYKTSLEELKTET 260 EIWEMENKGNNKKIDFDENYNILVAQIKEYFTKEIENYNNRIDNIIDKKELLKYSEEKEE 274 EIWKMENKGNNKKIDFDENYNILVAOIKEYFTKEIENYNNRIDNI IDKKELLKYSEKKEE 276

R-X4-H

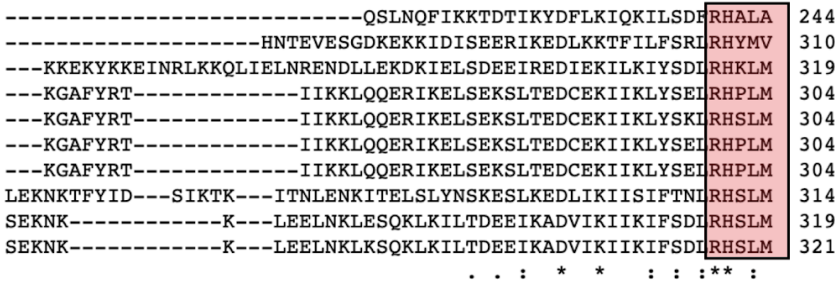

HEDFDFIOKFFDDELD-----------KNRFDISTISLIKTMLOEKEEKYYOEKNNYIEDS 294 $\therefore: *::: * *$ HYNYKFYENLYSGKNF IIYNKDKSKSRRF SELLDLNIFKELSK-IKLVKNRAVSNYLDKK 369 YNYQYFENLFENKKIS---KEKNEDVNLTELLDLNLFRYLPL-VRQLKLENKTNYLEKE 375 YDYQYFENLFENKEN----------SELTKNLNLDIFKSLPL-VRKMKLNNKVNYLEDN 353 YDYOYFENLFENKKN---------DDLMKDLNLDLFKSLPL-IRKMKLNNKVNYLEDG 353 HYDYQYFENLFENKEN----------SELTKNLNLDIFKSLPL-VRKMKLNNKVNYLEDN 353 HYDYQYFENLFENKEN----------SELTKNLNLDIFKSLPL-VRKMKLNNKVNYLEDN 353 AYDYKSFENLFENIEN---------EELKNLLDLNLFKSIRM-SDEFKTKNRTNYLDGT 363 HYEYKYFENLFENKKN----------EELAELLNLNLFKNLTL-LRQMKIENKTNYLEGD 368 GYEYKYFENLFENKKN----------EELAELLNLNLFKNLTL-LRQMKIENKTNYLEGR 370 *:::. : :::.. :.::: :

DTLTLFDEKESNFSKIHNFYIKISQKKPAFNKLINSFLSKDGVPNEELKSYLAT------- 348 TTIHVLNKN-INAI KLLDIYRDICETKNGFNNF INNMMT ISGEEDKEYKEMVTKHFNENM 428 DKITVLGVS-DSAIKYYSYYNFLCEQKNGFNNF INSFFSNDGEENKSFKEKINLSLEKEI 434 DTLFVLQKT-KKAKTLYQIYDALCEQKNGFNKF INDFFVSDGEENTVFKQIINEKFQSEI 412 DTLFVLQKT-KKAKTLYQIYDALCEQKNGFNKF INDFFVSDGEENTVFKQIINEKFQSEM 412 DTLFVLOKT-KKAKTLYQIYDALCEOKNGFNKF INDFFVSDGEENTVFKQI INEKFQSEM 412 DTLFVLQKT-KKAKTLYQIYDALCEQKNGFNKF INDFFVSDGEENTVFKQI INEKFQSEM 412 ESFTIVKKH-QNLKKLYTYYNNLCDKKNGFNTF INSFFVTDGIENTDFKNLIILHFEKEM 422 EKFNILGKD-VRAKNALGHYDLLVEOKNGFNNF INSFFVODGTENLEFKKFIDENF IKAO 427 EEFNI IGKN-IKAKEVLGHYNLLAEQKNGFNNF INSFFVQDGTENLEFKKLIDEHFVNAK 429

$$
\text { : : } \quad \text { *: : * .**.:*.: : .* : *.: }
$$


bioRxiv preprint doi: https://doi.org/10.1101/2022.01.21.477265; this version posted January 21, 2022. The copyright holder for this preprint (which was not certified by peer review) is the author/funder, who has granted bioRxiv a license to display the preprint in perpetuity. It is made available under aCC-BY-NC-ND 4.0 International license.

\section{C-terminal HEPN domain}

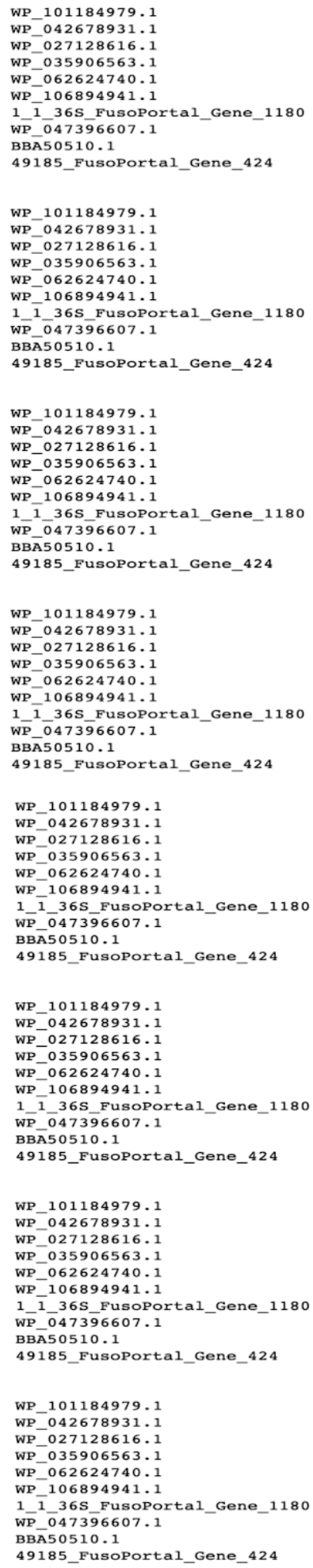

KLKEYFESTFIEKRFFHFCVKFFNKKTKKEETKQKNIFNLIENETLEELVKESPLLQIIT

RIIT 522 YLSKSLDRRDN--------RFK----KL--IKD-----YKFRDTEDIFCSDRENNLVKLYI 626 YLETYELKEEKE----KFNLK----KL--OET-----IOKTGEENWLLPQNKNNLFKFYL 607 YLTSFLKEEEKE----KFNLE----KM--OKI-----IOKTEEEDWLLPETKNNLFKFYL 607 YLTYFLKEEEKE----KFNLE----KM--OKI-----IOKTEEEDWLLPETKNNLFKFYL 607 YLTYFLKEEEKE----KFNLE----KM--OKI-----IOKTEEEDWLLPETKNNLFKFYL 607 YLNYTAPKNK--------FNFA----KL--EEELOKIOSTSEMGADYLNVSPENNLFKFYI 626 YLNYCMEKEEDGK-NYKFHFK----EI--S------EIKDTHNEEWLENTSENNLFKFYI 643 YLKYYKDK-EAQK-NYEFPFE----EI--F------ENKDTHNEEWLENTSENNLFKFYI 612 : $\quad$. *.: :

LLYLFIPKELQGEFVGFILKIYHHTKNITNDTKE------------DEKSIEDTQNSFSL 570 LMYILLPVEIRGDFLGFVKKNYYDLKHVDFIDKRNNDNK-----------------DTFFH 670 LTYILLPFEFRGDFLGFVKKHYYDIKNVEFLDENNDRLT--------PEQLEKMKNDSFFN $66 \mathbf{6}$ LTYLLLPYELKGDFLGFVKKHYYDIKNVDFMDENQSS-----------KIIESKEDDFYH 656 LTYLLLPYELKGDFLGFVKKHYYDIKNVDFIDENQNNIQ--------VSQTVEKQEDYFYH 660 LTYLLLPYELKGDFLGFVKKHYYDIKNVDFMDENQNNIQ--------VSQTVEKQEDYFYH 660 LTYLLLPYELKGDFLGFVKKHYYDIKNVDFMDENQNNIQ--------VSQTVEKQEDYFYH 660 LTYIMLPVEFKGDFLGFVKNHYYNIKNVDFMDESLLDENEVDSNKLNEKIENLKDSSFFN 686 LTYLLLPMEFKGDFLGVVKKHYYDIKNVDFTDESEKELS-------QEQIDKMIGDSFFH 696 LTYLLLPMEFKGDFLGVVKKHYYDIKNVDFTDESEKELS--------QVQLDKMIGDSFFH 665 DLRLFEKNVKRIEVTSYSISDGFLGKKSREKFGKELEKFIYK-----------NVSIA--- 71 KIRLFEKNSKKYDILKESILTSERIGKYFSLLNTGAKYFEY---------------- 70 KIRLFEKNTKKYEIVKYSIVPDKKLKQYFKDLGIDTK-KIRLFEKNKY KIRLFEKNTKY KIRIFEKNIKKYEIVKYSVST \begin{tabular}{l|l} 
KIRLFENIKK & $\mathbf{7 2}$
\end{tabular} KIRLFEKNTKRYEIIKYSILTSDEIKRYFRLLELDVPYFEYE---------------- 707 : : : : :**: : : : * :

--------------ISHNODEFDIHLVIPVIKYYINLNKLIGDFEIYALLTYADKNS PTNIDIKEFNKSLVILPMMKNYQIIFKLLNDIEI --------------GGEENRGIFNKNIIIPIFKYYQIVLKLYNDVELAMLLTLSESDE LILDOKSEVSGEKNKKVSLKNNGMFNKTILLFVFKYYQIAFKLFNDIELYSLFFLREKSC LTVEQKSEVSEEKNKKVSLKNNGMFNKTILLFVFKYYQIAFKLFNDIELYSLFFLREKSG LTVEQKSEVSEEKNKKVSLKNNGMFNKTILLFVFKYYQIAFKLFNDIELYSLFFLREKSE LTVEQKSEVSEEKNKKVSLKNNGMFNKTILLFVFKYYQIAFKLFNDIELYSLFFLREKSE ------------KSTDEIGIFNKNMILPIFKYYQNVFKLCNDIE I HALLALANKKO

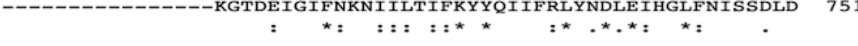

ITE----------KLSDITKRDDLKFRGYYNFSTLLFKTFM----------------------- 691

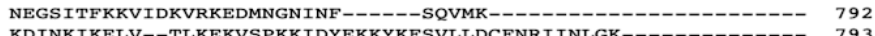

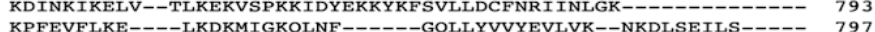
$\begin{array}{ll}\text { KPFEVFLKE----LKDKMIGKQLNF------GQLLYVVYEVLVK--NKDLSEILS----- } & 997 \\ \text { KPLEIFRKE----LESKMKDGYLNF-----GOLLYVVYEVLVK--NKDLDKIISS---- } & 823\end{array}$ KPFEVFLEE----LKDKMIGKOLNF------GQLLYVVYEVLVK--NKDLDKILS----- 823 KPFEVFLEE----LKDKMIGKQLNF------GQLLYVVYEVLVK--NKDLDKILS----- 823

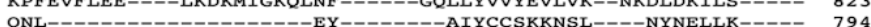
$\begin{array}{lll}\text { QNL--------1 } & 794 \\ \text { KILSDLK--------SYGNENINF------REFLYVIKQNNNSSTEEEYQKIWEKLESK } & 826\end{array}$ KILRDLK---------SYGNKNINF------REFLYVIKONNNSSTEEEYRKIWENLEAK 796

R-Xí-H

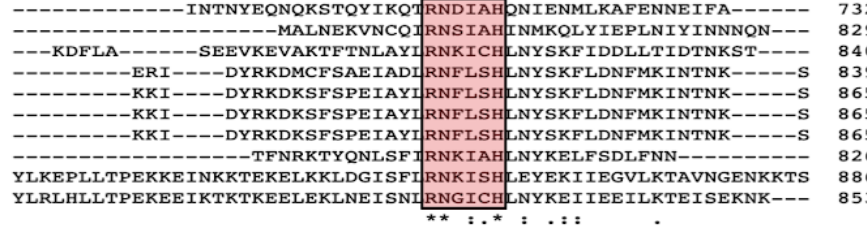

--

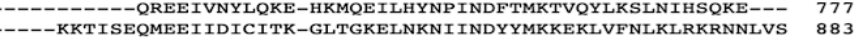
TDSEGKLLINDRIRKLIKFIRENNOKMNISIDYNYINDYYMKKEKFIFGQRKOAKTIIDS 900 DENKEVLIPSIKIQKMIKFIEECNLQSQIDFDFNFVNDFYMRKEKMFFIQLKQIFPDINS 899 DENKEVLIPSIKIQKMIQFIEKCNLQNQIDFDFNFVNDFYMRKEKMFFIQLKQIFPDINS 92

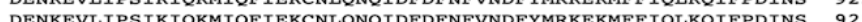
DENKE ETNADKVELNEKIKKIINEIKENE-LDKIELGFNEINDFEMKKOFMEGIKOVKEGNDTNADKVFLNEKIKKITNEIKENEL 943 $:$ : : : . $: *: \star \star: *:$ : :

--SKIAD----IHKKESLVPNDYYLIYKLKV--------7--------IELLKO---- 808 IDA------QQKNMKEKSILNKYDLNYKDENL------NIKEI---ILKVNDLNNKQ--- 925 GKKA------NKRNKAEELLKMYRVKKEN---------INLIYELSKKLNELTKSELFL 944 TEKQ----- KMNEKEEILRNRYHLTDKKNEQIKDEHEAQSQLYEKILSLQKIYSSD--- 950 TEKQ------KMNEKEEILRNRYHLTDKKNEQIKDEHEAQSQLYEKILSLQKIYSSD--- 976 TEKQ------KKSEKEEILRKRYHLINKKNEQIKDEHEAQSQLYEKILSLQKIFSCD--- 976 TEKQ------KKSEKEEILRKRYHLINKKNEQIKDEHEAQSQLYEKILSLQKIFSCD--- 976 KEKIIDGKRKQQNDSNNELLKKYGLSRTN--------IKDIFNKAWY---------- 919 $\begin{array}{ll}\text {-SDSITTERKRKEENNKRLKITYGLNYNN----------LSKIYEFSNTLREIVNSPLFL } & 992 \\ \text {-SDSITTTERERKEKNNKKLKETYELNCDN---------LSEFYETSNNLRERANSSSLL } & 954\end{array}$ SDSITTERERKEKNNKKLKETYEL

\section{i62 Figure S1. Multiple sequence alignment of Cas13c proteins. N-terminal and C-terminal HEPN i63 domains are highlighted as well as the R-X4-H motif.}




\begin{tabular}{|l|l|l|l|l|l|}
\hline Species & Strain & $\begin{array}{l}\text { CRISPR- } \\
\text { Cas } \\
\text { system } \\
\text { Type }\end{array}$ & Subtype & $\begin{array}{l}\text { Number of } \\
\text { spacers }\end{array}$ & Conservation \\
\hline F. nucleatum & 25586 & I & B1 & 15 & $0 \%$ \\
\hline F. nucleatum & 23726 & I & B1 & 23 & $0 \%$ \\
\hline F. periodonticum & $2 \_1 \_31$ & I, III & B1, A2 & $\begin{array}{l}\text { L1:42, L2:13, } \\
\text { L3:13, L4:28 }\end{array}$ & $\begin{array}{l}\text { L1:0\%, L2:0\%, } \\
\text { L3:0\%, L4:0\% }\end{array}$ \\
\hline F. varium & 27725 & I & B1 & $\begin{array}{l}\text { L1: 13, } \\
\text { L2:13, L3:24, } \\
\text { L4:18 }\end{array}$ & $\begin{array}{l}\text { L1: 9.75\%, } \\
\text { L4:2.04\% } \%\end{array}$ \\
\hline F. ulcerans & 49185 & I, VI & B1, C & $\begin{array}{l}\text { L1:17, L2:23, } \\
\text { L3:7 }\end{array}$ & $\begin{array}{l}\text { L1:0\%, L2:0\%, } \\
\text { L3:0\% }\end{array}$ \\
\hline F. mortiferum & 9817 & III & A1 & L1:4, L2:6 & L1:0\%, L2:0\% \\
\hline F. gonidiaformans & 25563 & I & B1 & 83 & $0 \%$ \\
\hline F. necrophorum & 1 1_36S & III, VI & A1, C & L1:13, L2:9 & L1:0\%, L2:0\% \\
\hline
\end{tabular}

Table S1. CRISPR-Cas system information of the eight analyzed Fusobacterium strains 


\begin{tabular}{|l|l|l|l|l|}
\hline Type & Cas proteins & Gene_FusoPortal_ID & GenBank_ID & Size (AA) \\
\hline I & Cas6 & 1183 & AVQ23192.1 & 250 \\
\hline I & Cas8a & 1184 & AVQ23193.1 & 518 \\
\hline I & Cas7 & 1185 & AVQ23194.1 & 300 \\
\hline$I$ & Cas5 & 1186 & AVQ23195.1 & 366 \\
\hline I & Cas3 & 1187 & AVQ23196.1 & 812 \\
\hline I & Cas4 & 1188 & AVQ23197.1 & 164 \\
\hline$I$ & Cas2 & 1190 & AVQ23199.1 & 92 \\
\hline$I$ & Cas1 & 1189 & AVQ23198.1 & 330 \\
\hline
\end{tabular}

Table S3. CRISPR-Cas cluster elements in F. nucleatum ATCC 23726. 
Table S6. CRISPR-Cas cluster elements in F. gonidiaformans 25563.

Table S5. CRISPR-Cas cluster elements in F. mortiferum 9817.

\begin{tabular}{|l|l|} 
GenBank_ID & Size (AA) \\
\hline AVQ29172.1 & 94 \\
\hline AVQ29173.1 & 333 \\
\hline AVQ29174.1 & 160 \\
\hline AVQ29175.1 & 861 \\
\hline AVQ29176.1 & 251 \\
\hline AVQ29177.1 & 314 \\
\hline AVQ29179.1 & 213 \\
\hline AVQ26923.1 & 1117 \\
\hline
\end{tabular}

\begin{tabular}{|l|l|l|l|l|}
\hline Type & Cas proteins & Gene_FusoPortal_ID & GenBank_ID & Size (AA) \\
\hline III & Cas6 & 2336 & AVQ19756.1 & 242 \\
\hline III & Csm5 & 2337 & AVQ19757.1 & 383 \\
\hline III & Csm4 & 2338 & AVQ19758.1 & 314 \\
\hline III & Csm3 & 2339 & AVQ19759.1 & 231 \\
\hline III & Csm2 & 2340 & AVQ19760.1 & 155 \\
\hline III & Cas10 & 2341 & AVQ19761.1 & 797 \\
\hline III & Cas2v7 & 2344 & AVQ19764.1 & 94 \\
\hline III & Cas2III8 & 2345 & AVQ19765.1 & 96 \\
\hline III & Cas1 & 2346 & AVQ19766.1 & 327 \\
\hline
\end{tabular}




\begin{tabular}{|l|l|l|l|l|}
\hline Type & Cas proteins & Gene_FusoPortal_ID & GenBank_ID & Size (AA) \\
\hline$I$ & Cas6 & 753 & AVQ16743.1 & 250 \\
\hline$I$ & Cas8a & 754 & AVQ16744.1 & 471 \\
\hline$I$ & Cas7 & 755 & AVQ16745.1 & 293 \\
\hline$I$ & Cas5 & 756 & AVQ16746.1 & 369 \\
\hline$I$ & Cas3 & 757 & AVQ16747.1 & 733 \\
\hline$I$ & Cas4 & 758 & AVQ16748.1 & 164 \\
\hline$I$ & Cas1 & 759 & AVQ16749.1 & 330 \\
\hline$I$ & Cas2 & 760 & AVQ16750.1 & 92 \\
\hline
\end{tabular}

Table S7. CRISPR-Cas cluster elements in F. periodonticum 2_1_31

\begin{tabular}{|l|l|l|l|l|}
\hline Type & Cas proteins & Gene_FusoPortal_ID & GenBank_ID & Size (AA) \\
\hline I & Cas2 & 316 & AVQ24518.1 & 92 \\
\hline I & Cas1 & 317 & AVQ24519.1 & 330 \\
\hline I & Cas4 & 318 & AVQ24520.1 & 164 \\
\hline I & Cas3 & 319 & AVQ24521.1 & 803 \\
\hline I & Cas5 & 320 & AVQ24522.1 & 359 \\
\hline I & Cas7 & 321 & AVQ24523.1 & 294 \\
\hline I & Cas8a & 322 & AVQ24524.1 & 514 \\
\hline I & Cas6 & 323 & AVQ24525.1 & 250 \\
\hline III & Cas2 & 1051 & AVQ25182.1 & 109 \\
\hline III & Cas1 & 1052 & AVQ25183.1 & 335 \\
\hline III & Csm6 & 1053 & AVQ25184.1 & 463 \\
\hline III & Cas6 & 1054 & AVQ25185.1 & 240 \\
\hline III & Csm5 & 1055 & AVQ25186.1 & 389 \\
\hline III & Csm4 & 1056 & AVQ25187.1 & 334 \\
\hline
\end{tabular}




\begin{tabular}{|l|l|l|l|l|}
\hline III & Csm3 & 1057 & AVQ25188.1 & 235 \\
\hline III & Csm2 & 1058 & AVQ25189.1 & 122 \\
\hline III & Cas10 & 1059 & AVQ25190.1 & 846 \\
\hline
\end{tabular}

Table S8. CRISPR-Cas cluster elements in F. necrophorum subsp. funduliforme 1_1_36S.

\begin{tabular}{|l|l|l|l|l|}
\hline Type & Cas proteins & Gene_FusoPortal_ID & GenBank_ID & Size (AA) \\
\hline II & Cas9 & 859 & AVQ20950.1 & 1337 \\
\hline II & Cas1 & 860 & AVQ20951.1 & 290 \\
\hline II & Cas2 & 861 & AVQ20952.1 & 106 \\
\hline II & Csn2 & 862 & AVQ20953.1 & 226 \\
\hline VI & Cas13c & 1180 & AVQ21256.1 & 1137 \\
\hline
\end{tabular}

Table S9. CRISPR-Cas cluster elements in F. varium 27725.

\begin{tabular}{|l|l|l|l|l|}
\hline Type & Cas proteins & Gene_FusoPortal_ID & GenBank_ID & Size (AA) \\
\hline I & Cas1 & 2395 & AVQ31955.1 & 333 \\
\hline$I$ & Cas2 & 2396 & AVQ31956.1 & 94 \\
\hline$I$ & Cas3 & 2393 & AVQ31953.1 & 858 \\
\hline$I$ & Cas4 & 2394 & AVQ31954.1 & 160 \\
\hline$I$ & Cas5 & 2392 & AVQ31952.1 & 250 \\
\hline$I$ & Cas6 & 2389 & AVQ31949.1 & 231 \\
\hline$I$ & Cas7 & 2391 & AVQ31951.1 & 314 \\
\hline
\end{tabular}




\section{REFERENCES}

1 Wu, C. et al. Forward Genetic Dissection of Biofilm Development by Fusobacterium nucleatum: Novel Functions of Cell Division Proteins FtsX and EnvC. mBio 9, doi:10.1128/mBio.00360-18 (2018).

2 Casasanta, M. A. et al. Fusobacterium nucleatum host-cell binding and invasion induces IL-8 and CXCL1 secretion that drives colorectal cancer cell migration. Sci Signal 13, doi:10.1126/scisignal.aba9157 (2020).

3 Kinder Haake, S., Yoder, S. \& Gerardo, S. H. Efficient gene transfer and targeted mutagenesis in Fusobacterium nucleatum. Plasmid 55, 27-38, doi:10.1016/j.plasmid.2005.06.002 (2006).

$4 \mathrm{He}$, X. et al. Interaction of $43 \mathrm{~K}$ OMP of Fusobacterium necrophorum with fibronectin mediates adhesion to bovine epithelial cells. Veterinary Microbiology, 109335, doi:10.1016/j.vetmic.2022.109335 (2022).

$5 \quad$ Zhang, Z. T. et al. Bacterial Genome Editing with CRISPR-Cas9: Taking Clostridium beijerinckii as an Example. Methods Mol Biol 1772, 297-325, doi:10.1007/978-1-4939-7795-6_17 (2018).

6 Nowak, C. M., Lawson, S., Zerez, M. \& Bleris, L. Guide RNA engineering for versatile Cas9 functionality. Nucleic Acids Res 44, 9555-9564, doi:10.1093/nar/gkw908 (2016).

7 Doudna, J. A. \& Charpentier, E. Genome editing. The new frontier of genome engineering with CRISPR-Cas9. Science 346, 1258096, doi:10.1126/science.1258096 (2014).

8 Barrangou, R. \& Marraffini, L. A. CRISPR-Cas systems: Prokaryotes upgrade to adaptive immunity. Mol Cell 54, 234-244, doi:10.1016/j.molcel.2014.03.011 (2014).

9 Karginov, F. V. \& Hannon, G. J. The CRISPR system: small RNA-guided defense in bacteria and archaea. Mol Cell 37, 7-19, doi:10.1016/j.molcel.2009.12.033 (2010).

10 Rath, D., Amlinger, L., Rath, A. \& Lundgren, M. The CRISPR-Cas immune system: biology, mechanisms and applications. Biochimie 117, 119-128, doi:10.1016/j.biochi.2015.03.025 (2015).

11 Makarova, K. S. et al. An updated evolutionary classification of CRISPR-Cas systems. Nat Rev Microbiol 13, 722-736, doi:10.1038/nrmicro3569 (2015).

12 Ponath, F. et al. RNA landscape of the emerging cancer-associated microbe Fusobacterium nucleatum. Nat Microbiol 6, 1007-1020, doi:10.1038/s41564-021-00927-7 (2021).

13 Zhang, Q. \& Ye, Y. Not all predicted CRISPR-Cas systems are equal: isolated cas genes and classes of CRISPR like elements. BMC Bioinformatics 18, 92, doi:10.1186/s12859-017-1512-4 (2017).

14 Couvin, D. et al. CRISPRCasFinder, an update of CRISRFinder, includes a portable version, enhanced performance and integrates search for Cas proteins. Nucleic Acids Res 46, W246-W251, doi:10.1093/nar/gky425 (2018).

15 Finn, R. D., Clements, J. \& Eddy, S. R. HMMER web server: interactive sequence similarity searching. Nucleic Acids Res 39, W29-37, doi:10.1093/nar/gkr367 (2011).

16 Snipen, L. \& Liland, K. H. micropan: an R-package for microbial pan-genomics. BMC Bioinformatics 16, 79, doi:10.1186/s12859-015-0517-0 (2015).

17 Wilkins, D. Draw Gene Arrow Maps in 'ggplot2' [R package gggenes version 0.4.0].

18 Kelley, L. A., Mezulis, S., Yates, C. M., Wass, M. N. \& Sternberg, M. J. The Phyre2 web portal for protein modeling, prediction and analysis. Nat Protoc 10, 845-858, doi:10.1038/nprot.2015.053 (2015).

19 Zadeh, J. N. et al. NUPACK: Analysis and design of nucleic acid systems. J Comput Chem 32, 170173, doi:10.1002/jcc.21596 (2011).

20 Silas, S. et al. Type III CRISPR-Cas systems can provide redundancy to counteract viral escape from type I systems. Elife 6, doi:10.7554/eLife.27601 (2017).

21 Liu, T. Y., Liu, J. J., Aditham, A. J., Nogales, E. \& Doudna, J. A. Target preference of Type III-A CRISPR-Cas complexes at the transcription bubble. Nat Commun 10, 3001, doi:10.1038/s41467019-10780-2 (2019). 
22 Vestergaard, G., Garrett, R. A. \& Shah, S. A. CRISPR adaptive immune systems of Archaea. RNA Biol 11, 156-167, doi:10.4161/rna.27990 (2014).

23 Wei, Y., Terns, R. M. \& Terns, M. P. Cas9 function and host genome sampling in Type II-A CRISPRCas adaptation. Genes Dev 29, 356-361, doi:10.1101/gad.257550.114 (2015).

24 Sanders, B. E., Umana, A., Lemkul, J. A. \& Slade, D. J. FusoPortal: an Interactive Repository of Hybrid MinION-Sequenced Fusobacterium Genomes Improves Gene Identification and Characterization. mSphere 3, doi:10.1128/mSphere.00228-18 (2018).

25 Briner, A. E. et al. Guide RNA functional modules direct Cas9 activity and orthogonality. Mol Cell 56, 333-339, doi:10.1016/j.molcel.2014.09.019 (2014).

26 Jinek, M. et al. Structures of Cas9 endonucleases reveal RNA-mediated conformational activation. Science 343, 1247997, doi:10.1126/science.1247997 (2014).

27 Mendoza, B. J. \& Trinh, C. T. In Silico Processing of the Complete CRISPR-Cas Spacer Space for Identification of PAM Sequences. Biotechnol J 13, e1700595, doi:10.1002/biot.201700595 (2018).

28 Shmakov, S. A. et al. The CRISPR Spacer Space Is Dominated by Sequences from SpeciesSpecific Mobilomes. mBio 8, doi:10.1128/mBio.01397-17 (2017).

29 Stano, M., Beke, G. \& Klucar, L. viruSITE-integrated database for viral genomics. Database (Oxford) 2016, doi:10.1093/database/baw162 (2016).

30 Shmakov, S. et al. Diversity and evolution of class 2 CRISPR-Cas systems. Nat Rev Microbiol 15, 169-182, doi:10.1038/nrmicro.2016.184 (2017).

31 Zhang, B. et al. Two HEPN domains dictate CRISPR RNA maturation and target cleavage in Cas13d. Nat Commun 10, 2544, doi:10.1038/s41467-019-10507-3 (2019).

32 Wang, J., Zhang, C. \& Feng, B. The rapidly advancing Class 2 CRISPR-Cas technologies: A customizable toolbox for molecular manipulations. J Cell Mol Med 24, 3256-3270, doi:10.1111/jcmm.15039 (2020).

33 Cox, D. B. T. et al. RNA editing with CRISPR-Cas13. Science 358, 1019-1027, doi:10.1126/science.aaq0180 (2017).

34 Todd, S. M., Settlage, R. E., Lahmers, K. K. \& Slade, D. J. Fusobacterium Genomics Using MinION and Illumina Sequencing Enables Genome Completion and Correction. mSphere 3, doi:10.1128/mSphere.00269-18 (2018).

35 Pickar-Oliver, A. et al. Targeted transcriptional modulation with type I CRISPR-Cas systems in human cells. Nat Biotechnol 37, 1493-1501, doi:10.1038/s41587-019-0235-7 (2019).

36 Watanabe, S. et al. Composition and Diversity of CRISPR-Cas13a Systems in the Genus Leptotrichia. Front Microbiol 10, 2838, doi:10.3389/fmicb.2019.02838 (2019).

37 Shah, S. A. et al. Comprehensive search for accessory proteins encoded with archaeal and bacterial type III CRISPR-cas gene cassettes reveals 39 new cas gene families. RNA Biol 16, 530-542, doi:10.1080/15476286.2018.1483685 (2019).

38 Bhaya, D., Davison, M. \& Barrangou, R. CRISPR-Cas systems in bacteria and archaea: versatile small RNAs for adaptive defense and regulation. Annu Rev Genet 45, 273-297, doi:10.1146/annurev-genet-110410-132430 (2011).

39 Cass, S. D. et al. The role of Cas8 in type I CRISPR interference. Biosci Rep 35, doi:10.1042/BSR20150043 (2015).

40 Richter, H. et al. Characterization of CRISPR RNA processing in Clostridium thermocellum and Methanococcus maripaludis. Nucleic Acids Res 40, 9887-9896, doi:10.1093/nar/gks737 (2012).

41 Haft, D. H., Selengut, J., Mongodin, E. F. \& Nelson, K. E. A guild of 45 CRISPR-associated (Cas) protein families and multiple CRISPR/Cas subtypes exist in prokaryotic genomes. PLoS Comput Biol 1, e60, doi:10.1371/journal.pcbi.0010060 (2005).

42 Niewoehner, O. \& Jinek, M. Structural basis for the endoribonuclease activity of the type III-A CRISPR-associated protein Csm6. RNA 22, 318-329, doi:10.1261/rna.054098.115 (2016).

43 Horlbeck, M. A. et al. Compact and highly active next-generation libraries for CRISPR-mediated gene repression and activation. Elife 5, doi:10.7554/eLife.19760 (2016). 